\title{
A clinical neurological, neurophysiological, and neuropsychological study of sheep farmers and dippers exposed to organophosphate pesticides
}

\author{
G A Jamal, S Hansen, A Pilkington, D Buchanan, R A Gillham, M Abdel-Azis, \\ P O O Julu, S F Al-Rawas, F Hurley, J P Ballantyne
}

See end of article for authors' affiliations .....................

Correspondence to: Dr G A Jamal, Department of Neurology, Central Middlesex Hospital, Acton Lane, London NW10 7NS, UK

Accepted 17 October 200

\begin{abstract}
Objectives: To classify clinical diseases of the subjects with abnormal indices of peripheral neuropathy identified in field studies of sheep farmers and dippers exposed to organophosphate pesticides. To explore what neuropsychological profiles, if any, may be associated with neurophysiological damage in these subjects.

Methods: A case-control study (79 subjects) nested within the cross sectional study (685 subjects) of sheep farmers from the field study. Three groups with no, possible, and probable or definite neuropathy according to field studies were recruited. Investigations comprised symptoms of neuropathy, neurologial signs, motor and sensory nerve conduction, electromyography, quantitative sensory testing, and neuropsychological tests.

Results: The incidence of clinical neuropathy increased from $7 \%$ in the no neuropathy group to $52 \%$ in the probable or definite neuropathy group based on nerve conduction measurements or presence of neurological signs. Sensory abnormalities were found more often than motor deficits. Small diameter nerve fibres were also affected more than large fibres.

Conclusions: The neuropathy is predominantly sensory and is characteristic of distal, chronic neuropathy with no acute features. Small fibre populations are affected more than large fibre populations. Increasing severity of neuropathy was associated with anxiety and depression as measured in the neuropsychological tests.
\end{abstract}

$\mathrm{R}$ ecent studies suggest that organophosphates (OPs) can produce long term effects which may be secondary to one or more acute toxic episodes or as a result of chronic long term exposure to low doses of OPs insufficient to produce acute symptoms.

There is general agreement that long term effects can result after one or more acute cholinergic episodes and epidemiological studies suggest various long term consequences of exposure. $^{1-4}$

The chronic effects on the peripheral and central nervous system of low level exposure, not producing any clinically detectable cholinergic features, are less extensively documented..$^{5}$ It is difficult to relate effects to specific agents when occupationally exposed workers may be potentially exposed to various pesticides and other agents. The phosphorylation of neuronal protein sites may contribute to the underlying disorder but genetic differences in detoxification of enzymes and non-specific binding are also known to account for some of the interindividual variation in susceptibility to anticholinesterases. ${ }^{5}$ Other enzyme systems or proteins may contribute to this effect. ${ }^{5}$

Many studies have been published on the effects of long term low level exposure and although most showed correlation between exposure and effect, the results have not been as consistent as those after acute poisoning episodes. ${ }^{5}$

For example, Stephens et al studied 158 sheep dippers and 155 quarry workers chosen as a control group. None of the farmers had experienced acute poisoning and had on average been involved in sheep dipping for 15 years. The authors found significant differences in sustained attention and speed of information processing in the exposed group but no effects on memory or learning. Basic neurological examinations, per- formed later on subgroups, showed some signs of sensory disturbance but little evidence of gross motor dysfunction in the farmers. ${ }^{7}$

Fiedler $e t$ al $^{8}$ reported on a neuropsychological study of 57 fruit tree sprayers and 42 non-exposed farmers or shopkeepers. Although the study found reduced reaction time in highly exposed people this was thought to be largely attributable to age, and there was no significant difference in other neurobehavioural tests.

Ames et al ${ }^{9}$ studied 45 agricultural pesticide applicators who used various organophosphates, and who each had at least one documented episode of cholinesterase inhibition but were asymptomatic. Serial digit test scores were better in the exposed group. However, the authors found that a higher level of exposure sufficient to cause acute poisoning changed these measures.

Stokes et $a l^{10}$ measured vibration sensation in 90 orchard sprayers and 68 matched controls. The sprayers had used at least five OPs over a mean of 20 years. Vibration thresholds were measured out of season and showed a $75 \%$ increase in the finger thresholds, which was significant $(p<0.01)$, but not in the foot thresholds.

Studies of neurobehavioural manifestations of OP intoxication are subject to methodological difficulties due to inadequate control groups, small study populations, and difficulty in ascertaining the exact nature of the exposure. ${ }^{4}$

A limited number of studies considering neurological health effects of long term low dose exposures to OPs have often only reported findings in small groups of subjects and

Abbreviations: OPs, organophosphates; QST, quantitative sensory test; $E M G$, electromyography; SFEMG, single fibre EMG 
Table 1 Classification of likelihood of neuropathy based on neurological symptoms and quantitative sensory testing (QST)

\begin{tabular}{lll}
\hline QST score & Symptom score & Classification \\
\hline 0 & $<1$ & No neuropathy \\
0 & $\geqslant 1$ & Possible neuropathy \\
$\geqslant 1$ & $<1$ & Possible neuropathy \\
$\geqslant 1$ & 1 or 1.5 & Probable neuropathy \\
$\geqslant 1$ & $\geqslant 2$ & Definite neuropathy \\
\hline
\end{tabular}

Symptom score, number of symptoms derived from responses to neuropathy symptoms questionnaire; QST score, number of thresholds outside normal limits for hot, cold, or vibration.

have relied on basic assessments of neurological function. The nature of the exposure varies considerably between different studies, as does the presence of significant co-exposures, and the availability of adequate personal protection. This makes it difficult to overview the findings of most published studies in a consistent manner. There is also the potential difficulty of interpreting non-specific symptoms relative to mixed exposures.

\section{MATERIALS AND METHODS \\ Background}

The third phase of the exposure-response epidemiological study investigating the effects of OP pesticides in sheep farmers and dippers was a case-control study of the neurological, neurophysiological, and neuropsychological status of cases with abnormal neurological indices, and controls with normal indices. The aim was to facilitate the clinical interpretation of the associations found in the second phase epidemiological study, ${ }^{11}$ refine any exposure-response relations found, and compare neuropsychological status with neurological status and exposure to OPs.

\section{Materials}

To understand the nature of the neuropathy which may result from exposure to OPs. The study group was restricted to sheep farmers. It is known that certain inherent neuropsychological characteristics might influence both choice of job and performance on specific tests which are not related to occupational exposures. It was therefore considered desirable to design out these potential occupational selection effects. The case-control study was nested within the cross sectional study of sheep farmers from the second phase study. ${ }^{11}$ The cross sectional study surveyed 609 farmers with sheep dipping experience, 109 farmers with no sheep dipping experience, and 107 ceramics workers in two areas of the United Kingdom. ${ }^{11}$ The cases are subjects with definite or probable neuropathy in the second phase study and control subjects are farmers exposed to OPs but almost certainly without neuropathy in second phase. An intermediate group with possible neuropathy was included.

The neuropathy status was determined from the outcome of neuropathy scores derived from the responses to the neuropathy questionnaire and sensory tests performed during the second phase epidemiological field study with predefined criteria. These were based on the Mayo Clinic recommendations ${ }^{12}$ and summarised in table 1 . Further details of rationale for the Mayo method can be found in the accompanying paper. ${ }^{13}$

A subset of the 685 subjects involved in the field study were invited to participate in the clinical study. The neuropathy scores from the field studies were calculated and on the basis of this information farmers were placed into the following categories: probable or definite neuropathy ( 48 subjects); possible neuropathy (516 subjects) ; or no neuropathy (125 subjects). The exceptionally high number in the possible group pointed to methodological problems. It arose because of high scores for abnormality in the second phase quantitative sensory test (QST) scores, notably cold sensory threshold, and to a lesser extent, vibration threshold. These scores in turn reflected an inconsistency between the QST field measurements, especially cold, and the clinical reference values from which abnormality was defined. The most likely explanation is the low ambient temperatures encountered during the fieldwork but other factors may also have contributed. These issues are described and discussed in more detail in a separate paper. ${ }^{13}$

To ensure a wide representation of disease status in the clinical study, the objective was to select people in equal numbers from the no, possible, and probable or definite groups. Because of small numbers, all 44 sheep farmers from the 48 people in the merged probable or definite category were invited to participate in the clinical studies. Eighty from the 125 no neuropathy group were selected at random. Selection from the possible group for the third phase study was based on the remaining 269 after removal of those who had only been categorised as possible due to abnormal cold thresholds. Eighty people were selected at random from this group of 269. However, the subjects selected did include a wide range of cold threshold responses. During the recruitment to the clinical studies, two subjects from the no and one from the probable or definite neuropathy group were excluded because they reported that they were taking medication or had been recently diagnosed with conditions which could confound the diagnosis of neuropathy leaving 78 and 43 subjects respectively.

Seventy nine sheep dippers attended the assessments at the Institute of Neurological Sciences, Glasgow. No ceramics workers were invited to attend the clinical study, and the few non-exposed farmers who were invited did not take part. These facts were not known to the team at the time of investigation.

The 79 subjects assessed were less than had been originally intended, but this was not due to non-response, rather to unexpected delays in starting the clinical work in the third phase. It was thought, however, that sufficient subjects attended across the range of neuropathy classification to allow informative results from the third phase.

Each person was sent a letter inviting them to participate in the clinical studies in Glasgow. They were given some indication of their results from the field study. It was anticipated that those who had normal results would be unwilling to travel and participate in the next phase of the study, although this proved not to be the case. Each letter contained a prepaid reply envelope and a reply slip, on which they could indicate whether they were willing to participate in the study or not. By tracking non-response rates it was possible to determine how many letters needed to be sent out for the possible and no neuropathy categories to ensure an adequate number of participants for the clinical studies.

When there had been no reply to the letter of invitation to take part in the clinical studies, people were followed up by phone about 2 weeks after the letters were sent. As with the earlier field survey, this often resulted in a positive response when farmers had the opportunity to discuss the survey in more detail. However, where farmers were unable to take time off due to limited cover for their farm duties or due to other reasons, the reason for inability to participate was recorded. Several farmers returned the reply slip sent out with the letter of invitation stating that they were unwilling to take part in the clinical studies. This group were not followed up further.

The hospital based study had been approved by the local ethics committee and all those participating gave their informed, written consent.

\section{Methods}

The study was carried out in the Neurophysiology Department at the Institute of Neurological Sciences, Glasgow. The 


\begin{tabular}{|c|c|c|c|c|}
\hline \multirow[b]{2}{*}{ Variable } & \multicolumn{3}{|c|}{ Neuropathy classification } & \multirow[b]{2}{*}{$\begin{array}{l}\text { Field study } \\
\text { group }\end{array}$} \\
\hline & None & Possible & $\begin{array}{l}\text { Probable or } \\
\text { definite }\end{array}$ & \\
\hline Attending (n) & 17 & 38 & 24 & \\
\hline Analysed (n) & 15 & 34 & 23 & 612 \\
\hline \multicolumn{5}{|l|}{ Sex: } \\
\hline Female & 2 & 5 & 3 & 88 \\
\hline$\%$ & 13 & 15 & 13 & 14 \\
\hline \multicolumn{5}{|l|}{ Age $(y)$ : } \\
\hline Mean & 38.8 & 46.2 & 46.1 & 45.1 \\
\hline SD & 13.3 & 10.6 & 11.0 & 13.4 \\
\hline Range & $20-66$ & $30-64$ & $22-62$ & $15-75$ \\
\hline \multicolumn{5}{|c|}{ Alcohol (units/week): } \\
\hline Mean & 9.0 & 10.4 & 8.4 & 8.2 \\
\hline SD & 6.7 & 8.5 & 9.8 & 10.2 \\
\hline Range & $0-20$ & $1-40$ & $0-38$ & $0-45$ \\
\hline \multicolumn{5}{|l|}{ Country: } \\
\hline Scotland & 10 & 28 & 13 & 344 \\
\hline$\%$ & 59 & 78 & 57 & 56 \\
\hline \multicolumn{5}{|c|}{ Cumulative exposure (OPEXP): } \\
\hline Mean & 1349 & 4364 & 1758 & 2400 \\
\hline SD & 1638 & 8011 & 1486 & 4280 \\
\hline Range & $10-6534$ & $68-44010$ & $12-6088$ & $0-48810$ \\
\hline
\end{tabular}

research team were blinded to the subjects, OP exposure, classification of neuropathy, and occupation. Observer bias was minimised by the use of five different investigators who performed different groups of tests and a trained nurse who acted as a "subject's friend". The nurse accompanied each subject throughout their visit and remained vigilant to the need to avoid information on exposure being passed onto the clinical team. The nurse was employed independently and given training by a member of the Institute of Occupational Medicine project team, who also screened questionnaires at intervals to ensure consistency of recording. The investigators were not aware of the results obtained by the other investigators on each person and were instructed not to ask the subject any questions relating to occupation or exposure.

The neuropathy questionnaire and quantitative sensory tests which were carried out during the field study ${ }^{11}$ were repeated in a hospital environment by specialist staff. Additional neurophysiological tests were selected to maximise the information about involvement of different parts of the peripheral nervous system in a reasonable time. Sensory and motor function, large and small nerve fibre function, and neuromuscular transmission were all covered. Therefore, the battery of the investigations was chosen to provide indices of the functional integrity of the whole peripheral nerve fibre population, and was based upon the Mayo Clinic recommendations. ${ }^{12}$ These assessments were carried out on the right side of the body unless the neuropathy questionnaire data for the person suggested the existence of a right sided injury.

Neuropsychological effects were assessed by investigating psychomotor performance, learning, and memory.

All assessments were performed between January 1998 and the end of May 1998 avoiding periods of peak use of pesticides. Based on experience from the second phase study, it was also a period when farmers were prepared to set aside time to participate in this type of study.

The assessment began by completion of the questionnaire, which detailed exposure during the interval since the farm visit. The subject was then allocated to the neurological assessment (2.5 hours) or the neuropsychological assessment ( 1.5 hours) for the remainder of the morning session. Subjects then completed the other half of the assessment in the afternoon. It was realised that fatigue may influence the results of the neuropsychological assessment and the time of day at which the assessment took place was recorded.

On completion of the assessment the farmers were informed that they would receive a brief report on the outcome of their investigations once analysis of the data from the clinical studies had been completed.

\section{Assessment of recent exposure}

A questionnaire on recent exposure to OPs was administered to all third phase participants by the nurse. The format used was similar to that of the second phase questionnaire on the history of exposure. Only one incident was recorded where the subject passed on information during the clinical assessments, which might have indicated their occupational group. This information related to an electric fence, and was not thought to have allowed distinction between farming groups, and so the subject was not excluded from the third phase

\section{Neurological symptoms}

The neurological symptom questionnaire was based on the Mayo Clinic neurological symptom questionnaire which was designed as part of a battery of tests to improve the diagnosis, and estimate the prevalence and severity of polyneuropathy. ${ }^{12}$ Our questionnaire therefore focused on symptoms occurring in the upper and lower limbs to detect possible chronic neurological effects which may be associated with exposure to organophosphates. Questions on cranial nerve involvement were excluded. A symptom, limited to one side or present for less than a month was ignored when the questionnaires were scored.

The questionnaire was the same as that used during the second phase epidemiological survey, ${ }^{11}$ but excluding details of occupation or details of relevant occupational exposure. This was to ensure that the Institute of Neurological Sciences survey team did not have access to information about the subject, which might bias their assessment of neurological status. A neurologist (GAJ) administered the questionnaire.

\section{Clinical assessment}

The clinical assessment was also based on the Mayo Clinic recommendations. ${ }^{12}$ The presence of neurological signs (reflexes, sensation, muscle power) was assessed in both upper and lower limbs. Muscle power was assessed by applying an 
Table 3 Neurological and neurophysiological findings (n (\%))

\begin{tabular}{|c|c|c|c|c|c|c|c|c|c|c|}
\hline Subject group & $\mathrm{n}$ & $\begin{array}{l}\text { Neurolo } \\
\text { signs }\end{array}$ & $\begin{array}{l}\text { caNerve } \\
\text { conduction }\end{array}$ & $\begin{array}{l}\text { Neurologice } \\
\text { signs or } \\
\text { nerve } \\
\text { conduction }\end{array}$ & EMG* & SFEMG & $\begin{array}{l}\text { Sensory } \\
\text { abnormalit }\end{array}$ & $\begin{array}{l}\text { Motor } \\
\text { tyabnorma }\end{array}$ & $\begin{array}{l}\text { Small fibre } \\
\text { ityabnormality }\end{array}$ & $\begin{array}{l}\text { Large fibre } \\
\text { tyabnormality }\end{array}$ \\
\hline No neuropathy & 15 & $0(0)$ & $1(7)$ & 1 (7) & $3(21)$ & $0(0)$ & $0(0)$ & $0(0)$ & $0(0)$ & $1(7)$ \\
\hline Possible neuropathy & 34 & $3(9)$ & $7(21)$ & $10(29)$ & $10(30)$ & $5(15)$ & $6(18)$ & 0 (0) & $26(76)$ & $7(21)$ \\
\hline $\begin{array}{l}\text { Probable or definite } \\
\text { neuropathy }\end{array}$ & 23 & $4(17)$ & $8(35)$ & $12(52)$ & $11(52)$ & $1(4)$ & $7(30)$ & $2(9)$ & 21 (91) & $7(30)$ \\
\hline Total & 72 & $7(10)$ & $16(22)$ & $23(32)$ & $24(35)$ & $6(8)$ & $13(18)$ & $2(3)$ & $47(65)$ & $15(21)$ \\
\hline
\end{tabular}

The subject group is according to third phase study classification (based on assessment of neurological symptoms and QST measurements in the clinic). Of the 79 subjects who came for clinical investigations, four were excluded from the analysis due to clinical history and a further three subjects with findings not consistent with a generalised neuropathy were omitted from the results.

*The number in group was 14,33, and 21 for no, possible, and probable or definite neuropathy respectively.

Neurological signs, clinical assessment of reflexes, sensation, and muscle power; nerve conduction, motor or sensory conduction in lower limbs; neurological signs or nerve conduction, combination of two previous columns (this is the most important outcome measure, the subjects included here have neuropathy in a clinical sense and would be reported as such to a referring GP or physician); EMG, needle electromyography of a muscle in the foot (EDB) (the results indicate chronic neurogenic changes); SFEMG, the small numbers indicate that there is only little abnormality in the neuromuscular transmission; sensory abnormality: abnormal sural (sensory) nerve conduction and one or more abnormal QST thresholds; motor abnormality, abnorma conduction in common peroneal nerve (motor); small fibre abnormality, abnormal hot or cold sensation threshold; large fibre abnormality, abnormal vibration sensation threshold or abnormal sural nerve conduction.

inverse MRC scale, where a score of zero signifies normality and a higher score signifies increasing abnormality. GAJ also performed these assessments.

Nerve conduction studies

Nerve conduction studies were carried out with standard techniques, ${ }^{14}$ normally on the right side. In motor conduction studies of median and common peroneal nerves, latency, peak to peak muscle action potential amplitude, nerve conduction velocity, and F wave latency and persistence were measured. In the sensory studies of median and sural nerves, peak latency, peak to peak amplitude, and nerve conduction velocity were measured. These studies were carried out by a neurophysiologist ( $\mathrm{SH})$.

\section{Electromyography (EMG) and single fibre EMG (SFEMG)}

These studies were carried out by a different neurophysiologist (AP). The EMG was recorded in the right extensor digitorum brevis, tibialis anterior, and extensor digiti communis with disposable concentric needle electrodes. This was scored taking into account the degree of occurrence of spontaneous potentials at rest and polyphasia of motor unit potentials. The score for each muscle was 0 if there was less than $15 \%$ polyphasia and no spontaneous activity, l with 15\%-35\% polyphasia or spontaneous activity, and 2 if the polyphasia was greater than $35 \%$ and there was profuse spontaneous activity. The outcome variables were a score for each muscle and sum of scores for the three muscles investigated. The amplitude of potentials at maximum voluntary contraction was also noted but was not used for scoring.

Stimulated SFEMG studies were performed on the right extensor digitorum communis muscle to investigate the integrity of the neuromuscular junction. The mean consecutive difference in interval between the stimulus and muscle fibre response for 100 stimuli was recorded for 10 different muscle fibres. ${ }^{15}$ The mean jitter from these 10 measurements as well as the percentage of abnormal jitter, and the mean percentage of blockings (the absence of a muscle fibre response to a nerve impulse) were noted.

\section{Quantitative sensory tests}

Hot and cold thermal thresholds from the dorsum of the foot were measured to test for small peripheral nerve fibre function ${ }^{16}$ and vibration threshold over the middle of the index metacarpal bone and first metatarsal bone to test the large peripheral nerve fibre function. ${ }^{17}$ The hot threshold tests the unmyelinated $\mathrm{C}$ fibres; the cold threshold tests the thinly myelinated A $\delta$ fibres, whereas the vibration threshold tests the thickly myelinated $A \beta$ fibres. ${ }^{18}$ The equipment used was the same as in the second phase field studies ${ }^{11}$ but the tests were performed by a neuroscientist (DB).

Each QST thresholds was compared with age dependent clinical reference values to indicate abnormality. The reference values for the thermal sensory tests were calculated from a sample of 68 healthy male and female volunteers aged between 16 and 76 years. ${ }^{19}$ For the vibration threshold, reference values were obtained from the handbook for the Somedic Vibrameter from a study of 100 subjects. A discussion of the choice of percentiles for defining abnormal tests and graphs of threshold limit values against age can also be found in a separate paper. ${ }^{13}$

\section{Neuropsychological investigations}

A battery of psychometric tests were administered by a neuropsychologist (RAG) and assessed general intelligence, psychomotor function, attention, memory, mood, and affect. An estimate of general intelligence, verbal IQ, was used as a control variable, and was measured with the national adult reading test.

The Cambridge neuropsychological test automated battery (CANTAB) was developed for the assessment of cognitive defects in humans with degenerative brain disease. It consists of a series of interrelated computerised tests of memory, attention, and higher brain function and is administered through a touch sensitive screen. Tests of attention (motor screening, reaction time, and matching to sample visual search) and memory (pattern recognition, spatial recognition, paired associate learning, and spatial span) were performed.

Also the Rey auditory verbal learning test was used as a test of verbal memory. Mood and affect were assessed by the general health questionnaire which evaluates psychiatric morbidity $^{20}$ and the hospital anxiety and depression scale. ${ }^{21}$

\section{Data analysis}

At weekly intervals, the forms on which results from the investigations were entered and the questionnaires were sent to the Institute of Occupational Medicine by one of the independent nursing staff. Data from the forms were key entered onto computer and validated. The data comparable with the second phase study were analysed further at the Institute of Occupational Medicine and the rest were returned to Institute of Neurological Sciences in the form of a spreadsheet at the end of the clinical investigations. This was validated against the forms, in particular for data entries marked as missing in the spreadsheet

Symptoms scores, QST scores, and neuropathy outcome scores were derived at the Institute of Occupational Medicine in an identical manner to the scoring scheme defined for the 
second phase study. The QST score depends on comparison with an age dependent threshold. The age used was that at the time of clinical examination. The scoring of the neuropathy questionnaire is not age dependent. Care was taken with missing data to avoid the generation of a false positive score for the section in which the data were missing.

The clinical data had been stored in such a way that descriptive statistics and comparisons of second and third phase data could be carried out without any results being influenced by knowledge of the person's exposure. The third phase scoring was also sent to Institute of Neurological Sciences to permit the derivation of classification profiles for other clinical data, and for neuropsychology outcomes.

Only after all validation and scoring was completed, both of neuropathy and exposure data, was the project team provided with the data which matched the history of occupational exposure to neurological data.

\section{RESULTS}

\section{Analysis of non-response and non-attendance}

Response rates were 27 out of 43 subjects (63\%) for the probable or definite, 37 out of 80 (46\%) for the possible, and 31 out of $78(40 \%)$ for the no neuropathy category. Response rates were better for those people who had been informed that some aspects of the initial assessment performed during the second phase study were outside the expected range for their age group but there was still a satisfactory response from those people who had no evidence of neuropathy. Travelling distance did not dissuade subjects from the Hereford and Worcester region in participating and the response rate from this region was reasonable compared with that from Scotland.

Of the 95 responders, 79 subjects attended the clinic. Nonattendance was partly due to unexpected delays in starting the clinical work and partly due to the fact that farmers were single handed or had limited back up and could not take time away from the farm.

\section{Study population}

With the same methods as in the second phase field study, each subject was assigned a diagnostic category corresponding to the likelihood of neuropathy. Table 2 describes the clinical study group broken down by category and also includes similar variables for the phase 2 study group. There was a roughly equal proportion of women in each category, close to the overall prevalence of $13 \%$. The possible and probable or definite groups were, on average, both 7 years older than the no group, consistent with the greater likelihood of neuropathy in these groups. There was a widespread of ages within each group, overall from 20 to 66 years. There was very little difference in average alcohol consumption across the three groups, close to the overall average of 9.5 units of alcohol a week, although this does not reflect the wide range of consumption, from 0 to 40 units per week, within the study group as a whole. The characteristics for the non-responders were similar.

Overall, $67 \%$ of those attending the clinic were Scottish sheep dippers. There was a higher prevalence of Scottish sheep dippers in the possible group $(78 \%)$ than in the other two groups. Only farmers with sheep dipping experience attended the clinic, the minimum number of days dipped being 5 days for any farmer in the study group.

Three people had the following conditions recorded on their second phase neuropathy questionnaires: rheumatoid arthritis, family history of high foot arches, and hypertension requiring medication, which was associated with hypotension. It was decided to err on the side of caution and exclude them from the analysis of the third phase study to limit possible confounding effects. A further four subjects were found to have a distribution of abnormalities of their neurophysiological variables not consistent with a generalised neuropathy.
Three had carpal tunnel syndrome and one subject had changes consistent with possible radiculopathy. These subjects, two each from of the no neuropathy and possible neuropathy groups, were excluded from further analysis leaving 72 subjects, 15, 34, and 23 from the no, possible and probable or definite neuropathy groups respectively.

\section{Organophosphate exposure}

There seemed to be differences in mean cumulative exposure across the three groups (table 2). However, it was the possible group that had the highest mean exposure based on the exposure index derived in the second phase of the study. ${ }^{11}$ Much of the reason for this was due to the presence of a few very highly exposed people in this group. There was very little difference in the exposures experienced in the no and probable or definite groups, which was not consistent with a hypothesis of increased likelihood of neuropathy with cumulative exposure.

\section{Classification of neuropathy}

For the analysis of the neurological and neurophysiological data the subjects have been divided into three different groups based on the neuropathy questionnaire results and QST thresholds from the hospital based investigation. The criteria used for groups 1, 2, 3, and 4 (no, possible, probable, and definite neuropathy) are identical to those used in the field study (table 1) but based on the investigations in the clinic. In common with analysis carried out for other data, groups 3 and 4 (probable and definite neuropathy) are combined.

The $95 \%$ confidence limits (95\% CIs) of normality for motor and sensory latency, nerve conduction velocity, and potential amplitude were from our own laboratory or from those published in handbooks ${ }^{22}$ with the effect of setting more severe criteria thus increasing the specificity. Decision criteria for overall abnormality are described later and these are based on the clinical experience that a toxic neuropathy is likely to affect the distal part of the lower limb first. Table 3 gives an overview over the number (and percentage) of subjects in each group who had a deficit.

\section{Neurological and neurophysiological outcomes Cranial signs}

No subject in any group had abnormal cranial signs indicating that there is no involvement of the cranial nerves.

\section{Reflexes}

Each subject was scored as having abnormal reflexes if: the ankle reflex was absent or the ankle reflex was reduced and any other distal reflex was reduced or absent bilaterally.

\section{Sensory signs}

Each subject was scored as having abnormal sensory signs if two or more modes of sensation were affected in the classic stocking-glove pattern.

\section{Muscle power}

Each subject was scored as having abnormal muscle power if there was any weakness in the distal part of the lower limb.

The outcome for neurological signs is shown in table 3. Each subject was considered to have significant signs if at least two out of the three signs (reflexes, sensory, and muscle power) were abnormal.

\section{Nerve conduction}

Sural nerve: sensory

The sensory function in the lower limb for each subject was scored abnormal if both sural potential latency and amplitude were abnormal. 


\begin{tabular}{lccccc}
$\begin{array}{l}\text { Table } 4 \\
\text { study }\end{array}$ & Symptoms (n (\%)): hospital based clinical \\
\hline Subject group & $\mathrm{n}$ & $\begin{array}{l}\text { Autonomic } \\
\text { symptoms* }\end{array}$ & $\begin{array}{l}\text { Sensory } \\
\text { symptoms }\end{array}$ & $\begin{array}{l}\text { Motor } \\
\text { symptoms }\end{array}$ \\
\hline No neuropathy & 15 & $3(20)$ & $0(0)$ & $0(0)$ \\
Possible neuropathy & 34 & $9(26)$ & $4(12)$ & $4(12)$ \\
$\begin{array}{l}\text { Probable or definite } \\
\text { neuropathy }\end{array}$ & 23 & $14(61)$ & $18(78)$ & $11(48)$ \\
Total & 72 & $26(36)$ & $22(31)$ & $15(21)$ \\
\hline
\end{tabular}

*Autonomic symptom scores of 0.5 or above.

Table 5 Subjects with abnormal QST (n (\%)): hospital based clinical study

\begin{tabular}{lcccc}
\hline Subject group & $\mathbf{n}$ & $\begin{array}{l}\text { Hot } \\
\text { threshold }\end{array}$ & $\begin{array}{l}\text { Cold } \\
\text { threshold }\end{array}$ & $\begin{array}{l}\text { Vibration } \\
\text { threshold }\end{array}$ \\
\hline No neuropathy & 15 & $0(0)$ & $0(0)$ & $0(0)$ \\
Possible neuropathy & 34 & $12(35)$ & $22(65)$ & $8(24)$ \\
$\begin{array}{l}\text { Probable or definite } \\
\text { neuropathy }\end{array}$ & 23 & $14(61)$ & $17(74)$ & $5(22)$ \\
Total & 72 & $26(36)$ & $37(62)$ & $13(18)$ \\
\hline
\end{tabular}

Table 6 Estimation of incidence of neuropathy in the farmer population studied in second phase

\begin{tabular}{|c|c|c|c|}
\hline Subject group & $\mathrm{n}$ & $\begin{array}{l}\text { Incidence of } \\
\text { neuropathy } \\
\text { in phase } 3\end{array}$ & $\begin{array}{l}\text { Estimated } \\
\text { number with } \\
\text { neuropathy in } \\
\text { phase } 2\end{array}$ \\
\hline No neuropathy & 125 & $1 / 15$ & 8 \\
\hline Cold QST abnormal only & 247 & $1 / 15$ & 16 \\
\hline Possible neuropathy & 269 & $10 / 34$ & 79 \\
\hline $\begin{array}{l}\text { Probable or definite } \\
\text { neuropathy }\end{array}$ & 44 & $12 / 23$ & 23 \\
\hline Total & 685 & - & $126(18 \%)$ \\
\hline
\end{tabular}

Incidence of neuropathy in general population is $0.2 \%-0.5 \%$.

\section{Common peroneal nerve: motor}

The motor function in the lower limb for each subject was scored abnormal if at least two out of the three action potential variables (latency, amplitude, or conduction velocity) was abnormal.

The outcome for nerve conduction is shown in table 3. Each subject was considered to have significant nerve conduction deficit if either sensory function or motor function in the lower limb was abnormal. This abnormality would be compatible with a toxic neuropathy.

\section{Combination of neurological signs and nerve conduction}

The number of subjects in each group with either neurological signs or abnormal nerve conduction are given in table 3. These subjects were considered to have neuropathy. There was no overlap between subjects that have neurological signs and abnormal nerve conduction with the criteria used. This does not mean that these deficits do not coexist, only that the criteria for the analysis were set rather strict. Out of 16 subjects with abnormal nerve conduction six $(38 \%)$ had one neurological sign.

\section{Electromyography}

Table 3 shows the number of subjects in each group with abnormal findings in the extensor digitorum brevis muscle. Four subjects had declined this investigation and the number in each group were reduced accordingly. Most of the abnormal scores were 1 and only one subject had a score of 2 . No fibrillation or other spontaneous activity was found in any of the recordings and the abnormalities point to a remodelling of the motor unit indicating an axonal type neuropathy with chronic changes.

\section{Single fibre EMG}

Six subjects had SFEMG abnormalities with five of those in the possible neuropathy group. None of the people classified as normal on symptoms and QST had abnormal SFEMG. Of those with SFEMG abnormalities, three had one or more signs and four also had an abnormal EMG.

\section{Sensory versus motor abnormalities}

Fifteen subjects had neurophysiological sensory abnormalities defined as abnormal sural conduction and one or more QST value(s). By contrast, only two subjects had abnormal motor nerve conduction and both were in the definite neuropathy group.

\section{Small versus large nerve fibre abnormalities}

A larger proportion of subjects had abnormal small nerve fibre function $(47 \%-65 \%$ of 72$)$, assessed by hot or cold sensation threshold, than had abnormal large fibre function (15\%-21\% of 72), assessed by vibration threshold or sural nerve function.

\section{Characterisation of neurological and neurophysiological deficit}

After exclusions, these analyses were based on 72 subjects as shown in table 3 . The classifications were based on symptoms and QST measured in the clinic (third phase) and not in the field (second phase). Table 3 gives further information about the type neuropathy encountered in the sheep dippers. A much higher proportion of subjects ( $18 \%$ of 72 ) had sensory abnormalities defined as abnormal sural conduction and one or more abnormal QST values whereas only 3\% of 72 had abnormal motor nerve conduction and both were in the definite neuropathy group (table 4). Abnormality of small nerve fibre function, assessed by hot or cold sensation threshold, was three times more common (65\% of 72 ) than abnormal large fibre function, assessed by vibration threshold or sural nerve function $(21 \%$ of 72$)$ (table 5$)$.

\section{Neuropsychological outcomes}

The neuropsychological results will be described in more detail in a separate paper but a resume is given here.

There were no significant differences in IQ between the three neuropathy groups. After adjustment for age and verbal IQ most psychological symptom rating scale variables showed significant differences among the three groups with probable or definite neuropathy group consistently significantly higher than the other two, inferring greater anxiety and depression. There was no evidence that whatever effect was responsible for the neurophysiological and psychological disturbance also produced memory impairment.

\section{DISCUSSION}

\section{Participation rates}

As expected, participation rates were better for those people who had been informed that some aspects of the initial assessment performed during the second phase were outside the expected range for their age group. However, there was still a satisfactory response from those people who had no evidence of neuropathy at the initial assessment. There was no evidence to suggest that the non-attendees differed in any other significant way from those attending.

It had been assumed that travelling to the Institute of Neurological Sciences in Glasgow for the clinical studies might be a factor in dissuading subjects from Hereford and Worcester to participate. Although this reason was cited by 
several subjects, it was not restricted to those from Hereford and Worcester and the response rate from this region was reasonable compared with that from Scotland.

\section{Steps taken to reduce bias}

The investigators at the Institute of Neurological Sciences were only given a subject number to identify people for the clinical studies and had no knowledge of neuropathy classification, exposure to OPs, or occupation. Nor were any of the five investigators aware of the results of the others on each person. The exposure-response relation was not examined until the third phase clinical examinations had been completed and the exposures calculated by the model from the first phase study. Only at this point was the matching of exposure and medical identifications carried out.

The ages of the possible and probable or definite groups are higher than for the no group. However, the age is not thought to be a significant confounder because $(a)$ age dependency of neurophysiological variables have been taken into consideration, and $(b)$ there is considerable overlap between the age range of the subjects with no neuropathy (20-63 years) and a range of 32-65 years for those classified as having neuropathy.

\section{Correlation between exposure to organophosphates and neuropathy}

The results did not show that cumulative exposure to OPs correlated with the presence of neuropathy, but the study was not designed to look at this question. Several people who acted as contractors or dipped on other farms locally had a high number of dipping days, which skewed the picture. Some of them used more mechanised dipping processes and so had less actual exposure. Sheep dippers who handled concentrate had three times the reported prevalence of symptoms than those who did not handle concentrate. Furthermore, the biological effects of OP compounds varied from person to person (see review by $\mathrm{Jamal}^{5}$ ).

\section{Characterisation of neurological deficit}

The neuropathy found in this study is different from the pattern of neuropathy found in the separate entity of OP induced delayed neuropathy (OPIDN). There is a predominant involvement of motor fibres in OPIDN thus presenting with paralysis and weakness as the main feature although sensory fibres can also be affected. By contrast, the neuropathy described here is predominantly of a sensory type both symptomatically and neurophysiologically and is characteristic of distal, chronic axonopathy with no acute features. Small fibre populations are affected more than large fibre populations. Autonomic nervous system symptoms were commonly reported by the no neuropathy and possible neuropathy groups, but less commonly by the probable neuropathy group. However, the methods used to assess the relative involvement of small versus large and sensory versus motor fibre populations may not have identical sensitivity and the apparent more frequent involvement of small fibre populations may be in part a reflection of the difference of the sensitivity of the methods.

Autonomic nervous system symptoms were reported more commonly than peripheral nervous system symptoms in the second phase study. This is also the case in the third phase study indicating that the autonomic nervous system may be more vulnerable than peripheral nerves.

Detection of neuropathy from the neuropathy questionnaire results and QST thresholds gives high sensitivity but may give low specificity ${ }^{23}$ and some false positive results may occur. When other neurophysiological variables and neurological signs are considered as well, the number of subjects confirmed as having a clinically detectable neuropathy will decrease but the decision is taken with higher probability of being certain of the diagnosis.

In general the results of the third phase neurological investigations are consistent with increased reporting of symptoms, minor sensory changes, and abnormal EMG results found in other studies of long term health effects associated with OPs. The pattern of the findings is comparable with those found in neuropathies induced by chemical intoxication-for example, with acrylamide, arsenic, and thallium. Distal axonal degeneration is the principal and earliest feature of OPIDN in both experimental animals ${ }^{24}$ and humans. ${ }^{25}$ In toxic neuropathies, including those related to organophosphate compounds, it is thought that the vulnerability of nerve fibres is related to axonal length whereby long axons, both motor and sensory, are thought to be more susceptible than shorter axons. ${ }^{26}$ This assumption has been primarily based on earlier morphological data and more recent electrophysiological, clinical, and morphological data suggest that all fibre populations are equally vulnerable ${ }^{27}$ and that sensory abnormalities are invariably present upon careful clinical examination. ${ }^{28}$

The percentage of abnormalities of all variables listed in table 3 increases from the no neuropathy group through to the probable or definite neuropathy group. This is predictable and justifies the finding from the neuropathy questionnaire and QST measurement. The more symptoms, the more likely that further evidence of neuropathy will be found.

In farmers generally, the estimated overall rates of confirmed (clinical) neuropathy is $18 \%$ when the third phase findings are reflected back into the second phase population (table 6). By comparison, the incidence of neuropathy in the general population is $0.2 \%-0.5 \%$

\section{Neuropsychology}

The primary purpose of performing neuropsychological evaluation of the subjects was to test the hypothesis that those people who were identified by neurological or neurophysiological examination as probable or definite neuropathy would also show signs of central nervous system damage.

Despite potential limitations of sample size and selection of tests neuropsychological evaluation does indicate a highly significant difference between the three groups and that the probable or definite group reports more psychological symptoms on the self reported scales. The scales are strongly intercorrelated, but two distinct dimensions at least, may be inferred-namely, anxiety and depression. There is also evidence of a graduated effect as the possible group tend to report more symptoms than the no neuropathy group.

The main conclusion is that among the group of subjects identified from the second phase of the study, there is a subgroup with evidence of damage to the peripheral nervous system, significant anxiety and depression, and possible slowing of processing speed, but no evidence of memory impairment.

Confounders not considered or allowed for in this study are stress, which may alter the permeability of the blood-brain barrier, genetic differences, difference in ability to detoxify compounds, and possible combinations of exposure with consequent synergetic effects. ${ }^{5}$ These factors might affect the biological dose received by a person, as well as that perceived as a measure of exposure.

\section{CONCLUSIONS}

The neuropathy described in the third phase is predominantly of a sensory type both clinically and neurophysiologically and is characteristic of a distal chronic type of neuropathy with no acute features. Small fibre populations are affected more than large fibre populations. The results of the additional tests (clinical examination and nerve conduction) therefore corroborate the other aspects of the Mayo Clinic methods in detecting a suspected toxic neuropathy in the clinical studies.

Increasing severity of neuropathy, as based on symptoms and sensory tests in the clinic was associated with anxiety and depression as measured in the neuropsychological tests. The results did not show that the neuropsychological findings were related to cumulative exposure to OPs, but it was 


\section{Main messages}

- Sheep dippers exposed to OP pesticides have a high incidence of neuropathy.

- The neuropathy is characteristic of a distal, chronic, and predominantly sensory type with no acute features.

- The results corroborate the usefulness of the modified Mayo Clinic methods (neuropathy questionnaire and quantitative sensory testing) in detecting suspected toxic neuropathy.

- Increasing severity of neuropathy, as based on symptoms and sensory tests in the clinic was associated with anxiety and depression as measured in the neuropsychological tests.

\section{Policy implications}

- The use of organophosphate compounds should be reviewed urgently and health and safety requirements should be evaluated. At the very least, extra care should be taken when using the products particularly handling the concentrate, and extra training should be given.

- People developing neurological symptoms should probably never be exposed to OP compounds again.

acknowledged that the study design would have limited power to examine such a relation anyway.

\section{ACKNOWLEDGEMENTS}

We gratefully acknowledge the help of all farmers, their families, and farm workers who took part in this study. We acknowledge the work and diligence of the nursing staff (Miss M MacKinnon and Miss M J Dolan) and clerical staff (Ms E Jackson) who assisted throughout the study at the Institute of Neurological Sciences, Glasgow. We also thank Ms Mags Parker, Mrs Marion Brebner, and Mrs Margaret Burnett of the Institute of Occupational Medicine for their administrative support during the project.

\section{Authors' affiliations}

G A Jamal, P O O Julu, S F Al-Rowas, Imperial College School of Medicine, London, UK

G A Jamal, M Abdel-Azis, P O O Julu, S F Al-Rawas, J P

Ballantyne, Department of Neurology, Institute of Neurological Sciences, South Glasgow University Hospitals NHS Trust, Glasgow, Scotland, UK S Hansen, Department of Clinical Physics, Institute of Neurological Sciences,

R A Gillham, Department of Neuropsychology, Institute of Neurological Sciences,

A Pilkington, D Buchanan, F Hurley, Institute of Occupational Medicine, Edinburgh, Scotland, UK

\section{REFERENCES}

1 Savage EP, Keefe TF, Mounce LM, et al. Chronic neurological sequelae of acute organophosphate pesticide poisoning. Arch Environ Health 1988;43:38-45

2 Rosenstock L, Keifer M, Daniell WE, et al. Chronic central nervous system effects of acute organophosphate pesticide intoxication. Lancet $1991 ; 338: 223-7$.
3 McConnell R, Keifer M, Rosenstock L. Elevated quantitative vibrotactile threshold among workers previously poisoned with methamidophos and other organophosphate pesticides. Am J Indust Med 1994;25:325-34.

4 Steenland K, Jenkins B, Ames RG, et al. Chronic neurological sequelae to organophosphate pesticide poisoning. Am J Public Health 1994;84:731-6.

5 Jamal GA. Neurological syndromes of organophosphorus compounds. Adverse Drug React Toxicol Rev 1997;16:133-70.

6 Stephens R, Spurgeon A, Calvert IA, et al. Neuropsychological effects of long-term exposure to organophosphates in sheep dip. Lancet 1995;345: 1135-9.

7 Beach J R, Spurgeon A, Stephens R, et al. Abnormalities on neurological examination among sheep farmers exposed to organophosphate pesticides. Occup Environ Med 1996;53:520-5

8 Fiedler N, Kipen H, Kelly-McNeil K, et al. Long-term use of organophosphates and neuropsychological performance. Am J Ind Med 1997;32:487-96.

9 Ames R, Steenland K, Jenkins B, et al. Chronic neurologic sequelae to cholinesterase inhibition among agricultural pesticide applicators. Arch Environ Health 1995:50:440-3.

10 Stokes L, Stark A, Marshall E, et al: Neurotoxicity among pesticide applicators exposed to organophosphates. Occup Environ Med 1995; 52:648-53.

11 Pilkington A, Buchanan D, Jamal GA, et al. An epidemiological study of the relations between exposure to organophosphate pesticides and indices of chronic peripheral neuropathy, and neuropsychological abnormalities in sheep farmers and dippers. Occup Environ Med 2001;58:702-10

12 Dyck PJ, Karnes JL, Daube J, et al. Clinical and neuropathological criteria for the diagnosis and staging of diabetic polyneuropathy. Brain 1985; 108:861-80.

13 Buchanan D, Jamal GA, Pilkington A, et al. Clinical validation of methods of diagnosis of neuropathy in a field study of UK sheep dippers. Occup Environ Med 2002;59:442-6.

14 Kimura J. Electrodiagnosis. In: Diseases of nerve and muscle: principles and practice, 2nd ed. Philadelphia: FA Davis, 1989.

15 Stålberg E, Tronteli J V. Single fiber electromyography: studies in healthy and diseased muscles, 2nd ed. New York: Raven Press, 1994.

16 Jamal GA, Hansen S, Weir Al, et al. An improved automated method for the measurement of thermal thresholds. 1. Normal subjects. J Neurol Neurosurg Psychiatry 1985;48:354-60

17 Jamal GA, Hansen S, Ballantyne JP. An automated system for quantification of the sense of vibration in man. J Physiol (Lond) 1991;438:340P

18 Jamal GA, Weir Al, Hansen S, et al. The neurophysiologic investigation of small fibre neuropathy. Muscle Nerve 1987; 10:537-45.

19 Hansen S, Jamal GA, Weir Al, et al. An instrument for measurement of thermal thresholds in man. Biosensors 1988;3:391-401.

20 Goldberg DP, Hillier VF. A scaled version of the general health questionnaire. Psychol Med 1979;9:139-45.

21 Snaith RP, Zigmond AS. Hospital anxiety and depression scale. Acta Psychiatr Scand 1983;67:361-70

22 Delisa JA, Lee JH, Barron EM, et al. Manual of nerve conduction velocity and clincial neurophysiology, 3rd ed. New York: Raven Press, 1994.

23 Jamal GA, Hansen S, Weir Al, et al. An improved automated method for the measurement of thermal thresholds. 2. Patients with peripheral neuropathy. J Neurol Neurosurg Psychiatry 1985;48:361-6.

24 Davis CS, Richardson RJ. Organophosphorus compounds. In: Spencer PS, Schaumburg HH, eds. Experimental and clinical neurotoxicology. Baltimore: Williams and Wilkins, 1980:527-44.

25 Lotti M, Becker CE, Aminoff M. Organophosphate polyneuropathy: pathogenesis and prevention. Neurology 1984;34:658-62.

26 Spencer PS, Schaumburg HH. Pathobiology of neurotoxic axonal degeneration. In: Waxman SG, ed. Physiology and pathophysiology of axons. New York: Raven Press, 1978:265-82

27 De Rojas TC, Goldstein BD. Lack of evidence for the size principle of selective vulnerability of axons in toxic neuropathies. Toxicol Appl Pharmacol 1990;104:47-58

28 Moen BE. Environmental and occupational toxins. Current Opinions in Neurology and Neurosurgery 1991;4:442-6. 